\section{The prevalence of cystoid macular oedema on optical coherence tomography in retinitis pigmentosa patients without cystic changes on fundus examination}

M Hajali and GA Fishman

\begin{abstract}
Purpose To determine the prevalence of cystoid macular oedema (CME) by optical coherence tomography $(\mathrm{OCT})$ in retinitis pigmentosa (RP) patients with no evidence of cystic macular lesions on fundus examination. Methods We included 63 RP patients with no evidence of cystic-appearing macular changes on fundus examination. All patients underwent a complete ocular examination including best-corrected visual acuity using an ETDRS (Early Treatment Diabetic Retinopathy Study) chart, intraocular pressure measurement, anterior segment examination, and a detailed fundus examination. On 50 of the 63 patients, Fourier-domain OCT was performed using the radial slicer protocol. An additional 13 of the 63 patients were scanned using the macular thickness protocol on a time-domain OCT unit. The diagnosis of CME was defined by the presence of hyporeflective lacunae with well-defined boundaries on at least two of the scans.

Results The mean age of patients included in the study was 36 years (range 9-71 years). Out of the 63 patients examined, 20 showed CME in at least one eye $(32 \%)$, whereas 11 patients showed CME in both eyes (18\%).

Conclusions Our findings demonstrate that a substantial number of RP patients with CME, as determined by OCT, may not show cystic changes by direct ophthalmoscopy or contact lens biomicroscopy. Knowledge of the high frequency for CME in such patients can serve to identify those who may be amenable to
\end{abstract}

current or future treatment strategies of their macular oedema and can potentially impact on future therapeutic trials where visual acuity is used as an outcome measure.

Eye (2009) 23, 915-919; doi:10.1038/eye.2008.110; published online 18 April 2008

Keywords: retinitis pigmentosa; macular oedema; OCT

\section{Introduction}

The presence of cystic-appearing lesions in the macula, presumed to represent cystoid macular oedema (CME), has been previously observed to occur in all genetic types of patients with retinitis pigmentosa (RP). ${ }^{1-4}$ The prevalence of 11-20\% macular oedema in RP patients has most commonly been reported using fluorescein angiography (FA) or optical coherence tomography (OCT). ${ }^{2,5-7}$

In future treatment trials where central visual acuity is used as an outcome measure, the presence of cystic macular lesions could represent a confounding variable for monitoring a therapeutic intervention aimed at primarily improving macular photoreceptor cell function. Further, identifying the presence of macular cysts will additionally serve to identify those RP patients who might benefit from the use of carbonic anhydrase inhibitors (CAI) or intravitreal steroid injections to reduce the degree of macular oedema and possibly improve central visual acuity. ${ }^{8-13}$
Department of

Ophthalmology and Visual Sciences, University of Illinois at Chicago, Chicago, IL, USA

Correspondence: GA Fishman, Department of Ophthalmology and Visual Sciences (MC 648), Room 3.85, Eye and Ear Infirmary, 1855 W Taylor Street, Chicago, IL 60612-7234, USA

Tel: + 1312996 8939;

Fax: + 13129961950 .

E-mail: gerafish@

uic.edu

Received: 7 February 2008 Accepted in revised form: 14 March 2008 Published online: 18 April 2008

The authors have no proprietary interest in this work 
To our knowledge, the prevalence of subclinical macular oedema or cystic changes in the macula of RP patients has not been well defined. The presence of CME in RP patients cannot always be detected clinically with contact lens biomicroscopy or direct ophthalmoscopy. ${ }^{7,14}$ Additionally, Hee et $a l^{15,16}$ reported that OCT is more sensitive than contact lens biomicroscopy to detect macular oedema in patients with diabetic retinopathy.

Therefore, in the present study, we sought to determine the prevalence of cystic-appearing macular lesions using current OCT technology in RP patients with no evidence of cystic macular changes or an atrophicappearing macular lesion on fundus examination.

\section{Materials and methods}

\section{Human subjects}

We reviewed the charts of all RP patients diagnosed by one of the authors (GAF) who were seen within the past 5 years and who had neither cystic nor atrophic-appearing macular changes on funduscopic examination. From a group of 98 RP patients, we were able to re-evaluate prospectively 55 patients who were asked to return for a re-examination of the macula and OCT testing for the presence of CME. In addition, we included all of the eight new RP patients who were seen by the authors between 20 June 2007 and 1 November 2007 and presented with no evidence of cystic or atrophicappearing macular lesions.

The diagnosis of RP was based on the patients' history of nyctalopia, variable degrees of peripheral field restriction, and markedly reduced or non-deductible ERG a- and b-wave amplitudes, in addition to ophthalmic findings, including characteristic fundus changes of bone spicule-like pigment clumping and attenuation of the retinal vessels. We excluded all syndromic forms of RP except for type II Usher syndrome. Additionally, we excluded all aphakic and pseudophakic patients and any with uveitis, diabetic retinopathy, or any other ocular or systemic disease that could cause RP-like fundus changes. We did not exclude those who had a clinically limited, glistening epiretinal macular membrane, nonspecific macular pigment mottling, and/or a blunted foveal reflex, as these findings may be observed in patients without cystic macular changes as well as those with cystic changes.

The total number of subjects included in this study was 63 (age range 9-71 years). All patients had a complete eye examination, including best-corrected visual acuity, using an Early Treatment Diabetic Retinopathy Study (ETDRS) chart (The Lighthouse, Long Island City, NY, USA), anterior segment examination by slit lamp biomicroscopy, Goldmann applanation tonometry to measure the intraocular pressure, and a dilated fundus examination by direct ophthalmoscopy, binocular indirect ophthalmoscopy, and 78-D non-contact lens. The fundus examination and a determination for the presence or absence of macular cysts clinically were performed before acquiring images by OCT. Informed consents were obtained from all subjects after an explanation of the nature and possible consequences of the study.

This project was conducted in the Department of Ophthalmology at the University of Illinois at Chicago; it was approved by an institutional review board and was performed in accordance with the tenets of the Declaration of Helsinki. We certify that all applicable institutional and governmental regulations concerning the ethical use of human volunteers were followed during this research.

\section{Optical coherence tomography}

On 50 of the 63 patients who were examined, Fourierdomain OCT (FD-OCT) was performed using an RTVue model RT100 (Optovue Inc., Fremont, CA, USA; software versions 1.2.6 and 2.0.3.2) by two experienced examiners. This OCT system has a high-speed acquisition time of 26000 A-scans per second and a high-depth resolution retinal scanner $(5 \mu \mathrm{m})$, which provides an accurate detail of retinal structure. Only images with good quality (signal strength index $>40$ ) were included in this study. The scanning protocol used on FD-OCT was the radial slicer that acquires simultaneously (within $0.27 \mathrm{~s}$ ) twelve 6-mm radial line scans through the centre of the fovea at $15^{\circ}$ intervals using internal fixation, where every radial scan consists of an average of 1024 A-scans.

Thirteen additional patients were scanned using a time-domain OCT, OCT3 commercial instrument (StratusOCT, software version 4.0.1; Carl Zeiss Meditec Inc., Dublin, CA, USA). The macular thickness protocol was used where six 6-mm radial scans were acquired on each eye at $30^{\circ}$ intervals centred on the patient's fovea. Each of these six lines was acquired individually and sequentially by the examiner while the patient was fixating on an internal target, and each line consisted of 512 A-scans. The accepted scans had signal intensity strengths of 5 or more, except for two patients where the accepted signal intensity was 4 .

The protocol used to define CME on OCT scans was the presence of cystoid spaces as small hyporeflective lacunae with well-defined boundaries on at least two views of the radial scans in the macular area.

Out of our cohort of 63 patients, we included only one eye from one of the patients owing to an inability to obtain OCT images on the other eye as a consequence of media opacity, a lack of proper fixation, and markedly 
reduced visual acuity. Another patient had a prosthetic eye, and so only one eye was included.

\section{Results}

The average age of the patients included in this study was 36 years (range 9-71 years). There were 32 male patients $(51 \%)$ and 31 female patients (49\%) who participated. The study group was comprised of 53 Caucasians (84\%), 8 African Americans (13\%), 1 Hispanic (1.5\%), and 1 Asian (1.5\%). The average best-corrected visual acuity for all patients was $0.35 \log$ MAR units (range -0.04 to 2.7 , equivalent to $20 / 20[+2]$ to hand motion on a Snellen acuity chart).

Out of the 63 patients with no cystic-appearing macular change on fundus examination, 20 patients showed CME on OCT testing in at least one eye (32\%), and 11 patients showed CME in both eyes (18\%). The modes of inheritance, the number and percentage of patients, and the age range of the patients in each genetic group are summarized in Table 1.

The pedigree analysis of the patients in this study showed that 15 patients had autosomal dominant (average age, 43 years), 8 patients had autosomal

Table 1 A summary of the number of patients included in each genetic subtype, the age range, and the number and percentage of patients with CME in each group

\begin{tabular}{|c|c|c|c|c|c|c|}
\hline \multirow[t]{2}{*}{$\begin{array}{l}\text { Genetic } \\
\text { type }\end{array}$} & \multirow[t]{2}{*}{$\begin{array}{c}\text { Patients } \\
\text { included (n) }\end{array}$} & \multirow[t]{2}{*}{$\begin{array}{c}\text { Age range } \\
\text { (years) }\end{array}$} & \multicolumn{2}{|c|}{$\begin{array}{c}\text { Patients with } \\
\text { CME in } \\
\text { both eyes }\end{array}$} & \multicolumn{2}{|c|}{$\begin{array}{l}\text { Patients with } \\
\text { CME in at } \\
\text { least one eye }\end{array}$} \\
\hline & & & $\mathrm{n}$ & $\%$ & $\mathrm{n}$ & $\%$ \\
\hline $\mathrm{AD}$ & 15 & $16-53$ & 3 & 20 & 5 & 33 \\
\hline AR & 8 & $13-67$ & 2 & 25 & 5 & 62 \\
\hline X-linked & 7 & $9-42$ & 0 & 0 & 0 & 0 \\
\hline Isolated & 15 & $20-65$ & 4 & 27 & 7 & 47 \\
\hline Usher II & 16 & $20-71$ & 1 & 6 & 2 & 13 \\
\hline Unknown & 2 & $14-32$ & 1 & 50 & 1 & 50 \\
\hline Total & 63 & $9-71$ & 11 & 18 & 20 & 32 \\
\hline
\end{tabular}

$\mathrm{AD}=$ autosomal dominant; $\mathrm{AR}=$ autosomal recessive.



Figure 1 An OCT image with cystic-appearing spaces in the left eye of an RP patient who showed no evidence of macular cystic changes by fundus examination. recessive (average age, 35 years), 7 patients had X-linked recessive (average age, 19 years), 15 patients had isolated disease (average age, 39 years), and 16 patients had Usher II (average age, 35 years). The inheritance pattern for two of the patients was not determined because one of them was adopted (14-year old), and in the other patient (32-year old), it was undetermined if autosomal dominant or $\mathrm{X}$-linked recessive was the actual inheritance because of the lack of a sufficient number of family members available for evaluation.

Figure 1 shows OCT images with cystic-appearing spaces in the left eye of an RP patient who showed no evident cystic changes by fundus examination. Figure 2 shows a fundus photograph from the left eye of the same patient whose OCT is shown in Figure 1.

\section{Discussion}

The presence of cystic lesions can be detected by ophthalmoscopy, contact lens biomicroscopy, FA, or OCT imaging. It was reported that CME could be easily detected by OCT independent of the angiographic degree of leakage, ${ }^{7}$ and that OCT is at least as sensitive as FA for identifying macular oedema. ${ }^{17,18}$ The advantage of OCT over FA is that it can discriminate the cross-sectional layered structure of the retina in vivo to evaluate more precisely the presence of CME in RP patients. ${ }^{19}$ Additionally, OCT serves as a useful, time-efficient, and non-invasive tool in monitoring the clinical course of macular oedema and the effectiveness of therapy for patients by providing reproducible measurements. ${ }^{18-20}$ OCT images are usually acquired more often on RP patients who are thought to have cystic changes on clinical examination, but less often as a screening for

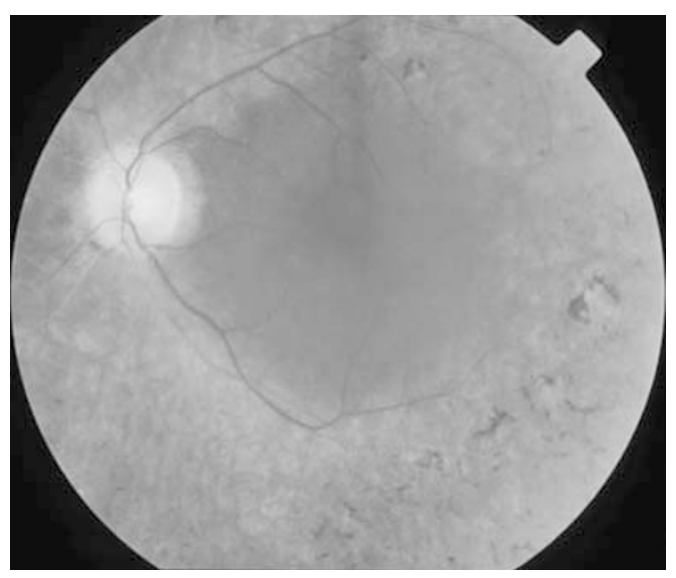

Figure 2 Fundus photograph from the left eye of the RP patient whose OCT image is shown in Figure 1. There are no evident cystic changes in the macula. The vertical shadow line represents a fixation target. 
CME on those who are not suspected as having macular oedema.

Hirakawa $e t \mathrm{al}^{7}$ found that in some eyes of patients with RP, mild CME demonstrated with OCT could not be detected by ophthalmoscopy or contact lens biomicroscopy. In a study of 39 patients with RP and $\mathrm{CME}$, Adackapara et $a l^{14}$ noted that OCT examination was able to detect macular cysts when clinical evaluation did not. Neither of these two reports ascertained their RP population for study based solely on the absence of CME on fundus examination. In the current study, we sought to determine the prevalence of cystic-appearing lesions in RP patients who showed no evidence of macular cystic changes on fundus examination. We used radial scans through the fovea to allow us to add to the sensitivity of OCT in the detection of cystic lesions in different meridians around the fovea rather than scanning only horizontally and vertically through the fovea.

The pathophysiology and aetiology of macular oedema in RP are not clearly understood. It was suggested to be the result of a breakdown in the blood-retinal barrier at the level of the retinal pigmented epithelium, perifoveal capillary plexus, or both. ${ }^{21-24}$ This barrier impairment is thought to be responsible for the release of retinal proteins into the circulation, which could be antigenic and trigger an autoimmune process contributing to the formation of CME and the presence of circulating antiretinal antibodies in RP patients. ${ }^{25,26}$

Different modes of therapy have been tried to resolve CME in RP patients. These include laser and surgical interventions, intravitreal steroid injections, systemic corticosteroids, and both topical and oral CAI. ${ }^{8-13}$ Since treatment options are available, it is of a significant value to determine the presence of CME so that treatment can be implemented in certain patients who may benefit from its use.

Several studies show that best-corrected visual acuity cannot be predicted from retinal thickness measurements at the fovea in RP patients with CME. ${ }^{7,18}$ However, the greater the width of the area covered by $\mathrm{CME}$, the greater will be the effect on the transmission of visual signals from the photoreceptors in the centre of the fovea, leading to a more extensive decrease in central vision. ${ }^{7}$ Nevertheless, one study found that both retinal thinning due to photoreceptor cell loss and retinal thickening due to presumed macular oedema were associated with lower visual acuity in patients with $\mathrm{RP} .^{27}$ In patients with macular oedema, visual acuity depends on several factors, including media opacity, the duration of $\mathrm{CME}$, and the degree of leakage. Visual acuity may not be appreciably reduced owing to a sparing of the fovea until a later course of the disease. ${ }^{3}$

One limitation of our study is that we may have underestimated the presence of macular oedema in our cohort of patients, as retinal thickness alone was not used as a criterion to define macular oedema on OCT scans. As funduscopically we did not attempt to evaluate macular thickness, we did not consider retinal thickness on OCT as a factor in our study. It is conceivable that not all instances of an increase in macular thickness result from the presence of non-cystic macular oedema rather than a possible secondary glial cell proliferation.

The proportion of RP patients with CME on OCT testing within each genetic group in this study was maximal for the autosomal-recessive group, followed by the isolated autosomal-dominant and Usher II groups. None of the seven patients in the X-linked group showed CME changes, even though this latter subtype is considered to show the most severe form of disease in terms of visual impairment. ${ }^{28} \mathrm{~A}$ previous study reported that macular oedema did not appear to be more common in any of the genetic groups. ${ }^{29}$ Because of the small number of patients in our cohort within the various genetic subtypes of RP, we did not report a statistical analysis for the difference in the presence of CME between genetic subtypes.

Our findings lead to the conclusion that even in the absence of cystic macular changes on fundus examination, it is necessary to screen RP patients by OCT to exclude the presence of CME. Our results document that as many as one-third of such patients will manifest cystic changes on OCT testing in at least one eye, whereas almost $20 \%$ will show such changes in both eyes. These observations have implications for future therapeutic trials where visual acuity is used as an outcome measure. Additionally, knowledge of the high frequency of macular oedema in these RP patients can also serve to identify those patients who may be amenable to current or future treatment strategies of their oedema.

\section{Acknowledgements}

This work was supported by funds from the Foundation Fighting Blindness, Owings Mills, MD, USA; Grant Healthcare Foundation, Lake Forest, IL, USA; NIH Core Grant EYOO1792; and an unrestricted departmental grant from Research to Prevent Blindness.

\section{References}

1 Fishman GA, Fishman M, Maggiano J. Macular lesions associated with retinitis pigmentosa. Arch Ophthalmol 1977; 95: 798-803.

2 Fishman GA, Maggiano JM, Fishman M. Foveal lesions seen in retinitis pigmentosa. Arch Ophthalmol 1977; 95: 1993-1996. 
3 Fetkenhour CL, Choromokos E, Weinstein J, Shoch D. Cystoid macular edema in retinitis pigmentosa. Trans Am Acad Ophthalmol Otolaryngol 1977; 83: 515-521.

4 Berson EL. Retinitis pigmentosa. The Friedenwald Lecture. Invest Ophthalmol Vis Sci 1993; 34: 1659-1676.

5 Pruett RC. Retinitis pigmentosa - clinical observations and correlations. Trans Am Ophthalmol Soc 1983; 81: 693-735.

6 Tranos PG, Wickremasinghe SS, Stangos NT, Topouzis F, Tsinopoulos I, Pavesio CE. Macular edema. Surv Ophthalmol 2004; 49: 470-490.

7 Hirakawa H, Iijima H, Gohdo T, Tsukahara S. Optical coherence tomography of cystoid macular edema associated with retinitis pigmentosa. Am J Ophthalmol 1999; 128: 185-191.

8 Cox SN, Hay E, Bird AC. Treatment of chronic macular edema with acetazolamide. Arch Ophthalmol 1988; 106: 1190-1195.

9 Grover S, Fishman GA, Fiscella RG, Adelman AE. Efficacy of dorzolamide hydrochloride in the management of chronic cystoid macular edema in patients with retinitis pigmentosa. Retina 1997; 17: 222-231.

10 Fishman GA, Gilbert LD, Anderson RJ, Marmor MF, Weleber RG, Viana MA. Effect of methazolamide on chronic macular edema in patients with retinitis pigmentosa. Ophthalmology 1994; 101: 687-693.

11 Fishman GA, Gilbert LD, Fiscella RG, Kimura AE, Jampol LM. Acetazolamide for treatment of chronic macular edema in retinitis pigmentosa. Arch Ophthalmol 1989; 107: $1445-1452$.

12 Ozdemir H, Karacorlu M, Karacorlu S. Intravitreal triamcinolone acetonide for treatment of cystoid macular oedema in patients with retinitis pigmentosa. Acta Ophthalmol Scand 2005; 83: 248-251.

13 Scorolli L, Morara M, Meduri A, Bacchi Reggiani L, Ferreri $\mathrm{G}$, Scalici SZ et al. Treatment of cystoid macular edema in retinitis pigmentosa with intravitreal triamcinolone. Arch Ophthalmol 2007; 125: 759-764.

14 Adackapara CA, Sunness JS, Dibernardo CW, Melia M, Dagnelie G. Prevalence of cystoid macular edema and stability in OCT retinal thickness in eyes with retinitis pigmentosa during a 48-week lutein trial. Retina 2008; 28: 103-110.

15 Hee MR, Puliafito CA, Wong C, Duker JS, Reichel E, Rutledge B et al. Quantitative assessment of macular edema with optical coherence tomography. Arch Ophthalmol 1995; 113: 1019-1029.

16 Hee MR, Puliafito CA, Duker JS, Reichel E, Coker JG, Wilkins JR et al. Topography of diabetic macular edema with optical coherence tomography. Ophthalmology 1998; 105: 360-370.

17 Stanga PE, Downes SM, Ahuja RM, Chong NHV, Antcliff R, Reck AC et al. Comparison of optical coherence tomography and fluorescein angiography in assessing macular edema in retinal dystrophies: preliminary results. Int Ophthalmol 2001; 23: 321-325.

18 Catier A, Tadayoni R, Paques M, Erginay A, Haouchine B, Gaudric A et al. Characterization of macular edema from various etiologies by optical coherence tomography. $A m$ J Ophthalmol 2005; 140: 200-206.

19 Hee MR, Izatt JA, Swanson EA, Huang D, Schuman JS, Lin $\mathrm{CP}$ et al. Optical coherence tomography of the human retina. Arch Ophthalmol 1995; 113: 325-332.

20 Chung H, Hwang JU, Kim JG, Yoon YH. Optical coherence tomography in the diagnosis and monitoring of cystoid macular edema in patients with retinitis pigmentosa. Retina 2006; 26: 922-927.

21 Cunha-Vaz JG, Travassos A. Breakdown of the blood-retinal barriers and cystoid macular edema. Surv Ophthalmol 1984; 28: $485-492$.

22 Vinores SA, Küchle M, Derevjanik NL, Henderer JD, Mahlow J, Green WR et al. Blood-retinal barrier breakdown in retinitis pigmentosa: light and electron microscopic immunolocalization. Histol Histopathol 1995; 10: 913-923.

23 Kuchle M, Nguyen NX, Martus P, Freissler K, Schalnus R. Aqueous flare in retinitis pigmentosa. Graefes Arch Clin Exp Ophthalmol 1998; 236: 426-433.

24 Newsome DA. Retinal fluorescein leakage in retinitis pigmentosa. Am J Ophthalmol 1986; 101: 354-360.

25 Heckenlively JR, Jordan BL, Aptsiauri N. Association of antiretinal antibodies and cystoid macular edema in patients with retinitis pigmentosa. Am J Ophthalmol 1999; 127: 565-573.

26 Heckenlively JR, Solish AM, Chant SM, Meyers-Elliott RH. Autoimmunity in hereditary retinal degenerations. II. Clinical studies: antiretinal antibodies and fluorescein angiogram findings. Br J Ophthalmol 1985; 69: 758-764.

27 Sandberg MA, Brockhurst RJ, Gaudio A, Berson EL. The association between visual acuity and central retinal thickness in retinitis pigmentosa. Invest Ophthalmol Vis Sci 2005; 46: 3349-3354.

28 Fishman GA, Farber MD, Derlacki AJ. X-linked retinitis pigmentosa. Arch Ophthalmol 1988; 106: 369-375.

29 Spalton DJ, Bird AJ, Clearly PI. Retinitis pigmentosa and retinal oedema. Br J Ophthalmol 1978; 62: 174-182. 